\title{
Joint energy and reserve dispatch in a multi-area competitive market using time-varying differential evolution
}

\author{
Kirti Pal ${ }^{1}$, Manjaree Pandit ${ }^{2 *}$, Laxmi Srivastava ${ }^{2}$ \\ ${ }^{I}$ Department of Electrical Engineering, Radha Govind Group of Institutions Meerut, INDIA \\ ${ }^{2}$ Department of Electrical Engineering,Madhav Institute of Technology and Science Gwalior, INDIA \\ "Corresponding Author: e-mail: manjaree_p@hotmail.com, Tel +91-0751-2665962,+91-0751-2409380
}

\begin{abstract}
In a deregulated electricity industry, competitive bidding for energy and reserve is increasingly gaining importance and playing a decisive role in maintaining system reliability. The transmission capacity limits have a significant impact on market cost, therefore these limits should also be considered while optimizing the joint dispatch. In this paper, a detailed comparison of classical differential evolution (DE) mutation strategies is carried out to study the role of tuning parameters. For avoiding the time and labor lost in tuning DE parameters, an improved DE algorithm with time varying chaotic mutation and crossover is proposed for solving the multi-product, multi-area market dispatch problem with physical and operational constraints. The proposed approach effectively handles the complex constraints like reserve requirements, zonal power balance constraints, area spinning reserve constraints, tie-line constraints and reserve and capacity coupling constraints. The efficiency and accuracy of the proposed algorithm is tested on two different test systems and is found to be better than classical DE in terms of convergence behavior and solution quality.
\end{abstract}

Keywords: Joint energy and reserve dispatch (JERD), Multi-area competitive electricity market, Reserve cost, Energy cost, Total market cost, Time-varying Differential evolution.

\section{Introduction}

Many countries have implemented deregulation of the electricity industries to create market competition for improving the economical efficiency of power system operation. In the competitive environment, it is necessary to maintain an adequate level of supply reliability to keep a secure operation. A real time market in energy can provide a balance between generation and demand whereas a real time reserve market is required to maintain this balance in case of a contingency. The operation of a real time market is managed by an independent system operator (ISO) who coordinates various activities to balance reliability and security with economics. In a competitive environment, several new concepts are applied to the traditional economic dispatch (ED). Commercial interest can affect the generator characteristics i.e. the generators may change their bid prices for improving their profit. Spinning reserve (SR) is one of the most important ancillary services (AS) which are needed for satisfying reliability requirements, including the desired level of security in power systems. By definition, spinning reserve is the unloaded section of synchronized generation that is able to respond immediately to serve load. Therefore it is desirable to optimize not only the generator bid prices for energy but also to provide AS (Shahidehpour et al, 2001).

In the competitive electricity market, one of the major concerns of the system operator is to achieve the economic efficiency while maintaining the system security. Ancillary services are provided to maintain system reliability and security. Based on the current practices, ancillary services can be either dispatched with energy dispatch simultaneously or dispatched sequentially. This paper presents the multi-area joint energy and reserve dispatch (JERD) employing an improved differential evolution approach.

Transmission limits play a very important role in the multi-area joint energy and reserve dispatching problem. If energy and reserve are dispatched without considering transmission limits, it is possible that larger amounts of energy and reserve be allocated to some units having lower prices. Now even though the units have the capacity to produce these amounts of energy and reserve, 
there may be bottlenecks in supply due to transmission limits. Therefore, in JERD while energy cost and reserve costs are minimized, the capability of transmission lines to deliver these to the consumer also needs to be considered.

The problem of joint energy and ancillary services dispatch in a multi-area competitive market is more complex than the traditional power dispatch. Many different methods have been proposed to solve the problem of joint energy and reserve dispatch (Cheung et al, 1999, Ma et al, 1999, Chen et al, 2005, Ma et al, 1998, Bakirtzis et al, 1998, Deventer et al, 2010, Rashidinejad et al, 2002). In (Cheung et al, 1999) the functional requirements of the energy and ancillary service dispatch for the interim ISO-NE electricity market are presented. An LP-based approach is presented in (Ma et al,1999) for solving the energy and auxiliary services multi-zone physical market dispatch problem where various constraints, such as reserve requirement constraints, network security limits, and sharing of constrained resource capacity have been considered. A hybrid approach combining sequential dispatch with a direct search method is developed for the multi-product, multi-area electricity market dispatch problem to facilitate economic sharing of generation and reserve across areas and to minimize the total market cost in a multi-area competitive electricity market. The effects of tie line congestion and area spinning reserve requirement are also consistently reflected in the marginal price in each area (Chen et al,2005). The complex mutual dependence of energy and AS dispatch and the growing importance of AS in competitive bidding in pool market is formulated in (Ma et al,1998, Bakirtzis et al,1998). The analysis of cost-effective dispatching of emergency reserves with a specific focus on supply and demand side options is presented in (Deventer et al , 2010). A flexible joint reserve and energy dispatch approach where the contingency reserve capacity is procured in advance and incremental energy is dispatched in response to meet real time demand-supply imbalances, is proposed in (Rashidinejad et al, 2002).

The multi-area JERD problem for a competitive power market is a more complex optimization problem than the conventional economic dispatch due to the various additional constraints such as i)area power balance constraints, ii) area spinning reserve constraints, iii) tie-line constraints and iv) constraints due to mutual dependency of energy and reserve. For such problems evolutionary computational techniques such as particle swarm optimization (Azadani et al, 2010), genetic algorithm (Xu et al, 2003, Wen et al, 2002), memetic algorithm (Hazrati et al, 2007) and harmony search (HS) (Paqaleh et al, 2009) have been applied very effectively. A new approach based on constrained particle swarm optimization is presented for the multi-product and multiarea electricity market dispatch problem where constraint handling is based on particle ranking and uniform distribution (Azadani et al, 2010).

Some interesting studies have been carried out on competitive electricity markets, for energy and reserve using probabilistic analysis (Bouffard et al, 2004), reliability criterion (Flynn et al, 2001) and multiobjective formulations for improving system security indices along with generator offer costs (Amjady et al, 2009).

A method using a recurrent neural network for considering generating unit reliability in the scheduling process which rewards units with lower forced outage probabilities has been proposed in ref. (Flynn et al, 2001). Reserve costs have been included explicitly in the cost function which results in reduced overall costs. A probabilistic method for a pool-based combined energy and reserve market, which considers both generators and interruptible loads is proposed in ref. (Bai et al, 2006).

A Genetic Algorithm based approach is presented in (Xu et al, 2003) for the design of an integrated market for energy and spinning reserve service with special focus on coordinated dispatch of bulk power and spinning reserve services. In (Wen et al, 2002), two different bidding schemes using genetic algorithm are proposed for building optimally coordinated bidding strategies for competitive suppliers in day-ahead energy and spinning reserve markets. In (Hazrati et al, 2007) presents a memetic algorithm approach for SR allocation considering ramp-rate constraints and investigates the impact of these constraints on allocation strategy associated with clearing prices. A hybrid harmony search optimization method is proposed for the joint dispatch of energy and reserve considering the bottling of reserve due to transmission limits (Paqaleh et al, 2009).

Though evolutionary methods do not always guarantee global best solutions, they usually attain a fast and near global optimal solution. It has been observed by researchers that all these methods very quickly find a good local solution but get stuck there for a number of iterations causing premature convergence. The major issue with evolutionary techniques is to maintain a proper balance between exploration (global search) and exploitation (local search). The issues which have emerged prominently in recent publications are i) Premature convergence, ii) setting up optimal tuning parameters and ii) finding optimal population size (Chaturvedi et al, 2009, Chiou et al, 2009, Storn et al,1995, Coelho et al,2009, Ratnaweera et al,2004, Sharma et al,2010, Sharma et al, 2010). Tuning parameters play a very significant role in obtaining convergence in these techniques. Optimal values of tuning parameters produce a proper balance between local and global search to get the best solution. There is a need to explore the role of different tuning parameters in various evolutionary computational techniques. Time varying acceleration coefficients (PSO_TVAC) (Chaturvedi et al, 2009, Ratnaweera et al,2004) were employed for iteratively controlling the global and local search components in PSO. The concept of variable scaling factor based on the one-fifth success rule of evolutionary strategies is employed in (Chiou et al, 2009)

This paper evaluates the effectiveness of all the basic DE strategies in producing feasible solutions for multi-area JERD problem. The paper presents a comparative analysis of i) the various DE mutation strategies ii) impact of tuning parameters and iii) proposes an improved time varying DE algorithm for the joint dispatch problem. The solution quality of DE variants is also compared with the PSO_TVAC strategy on two test systems and is found to be superior/or comparable. 
The major contributions of the paper are:

1. An alternative approach which is comparatively new and based on evolutionary optimization is successfully applied for JERD problem in competitive environment with multiple complex constraints.

2. The sectionalized block pricing for energy and reserve is implemented and studies are carried out for different reserve requirements, different tie-line limits and different loads.

3. A detailed study of classical DE mutation strategies is carried out for the JERD problem, their deficiencies have been brought out and then modifications proposed using time varying mutation.

4. Three DE variants with time varying mutation and crossover rates are proposed. All three variants are found to perform well; feasible and matching results (Nasr Hamdani et al, 2010) are achieved while all constraints are satisfied.

5. The JERD problem is quite complex compared to the classical ED (economic dispatch) due to the large number of constraints, like the reserve limits and energy-reserve coupling constraints. Due to multi-area operation, lie-line constraints too affect the total cost significantly.

6. The paper focuses on JERD for a given demand, tie-limit and reserve required and evaluates the optimal dispatch of energy and reserve while satisfying all the operational constraints.

\section{Problem formulation for joint energy and reserve dispatch}

The objective of JERD in a multi-area electricity market is to minimize the total cost of energy and reserve while considering various constraints such as area power balance, area spinning reserve, transmission capacity limits and constrained resource capacity shared between generation and reserve. If inelastic load is assumed, then cost minimization instead of profit maximization can be considered. Figure 1 shows a simple representation of JERD problem for two areas.

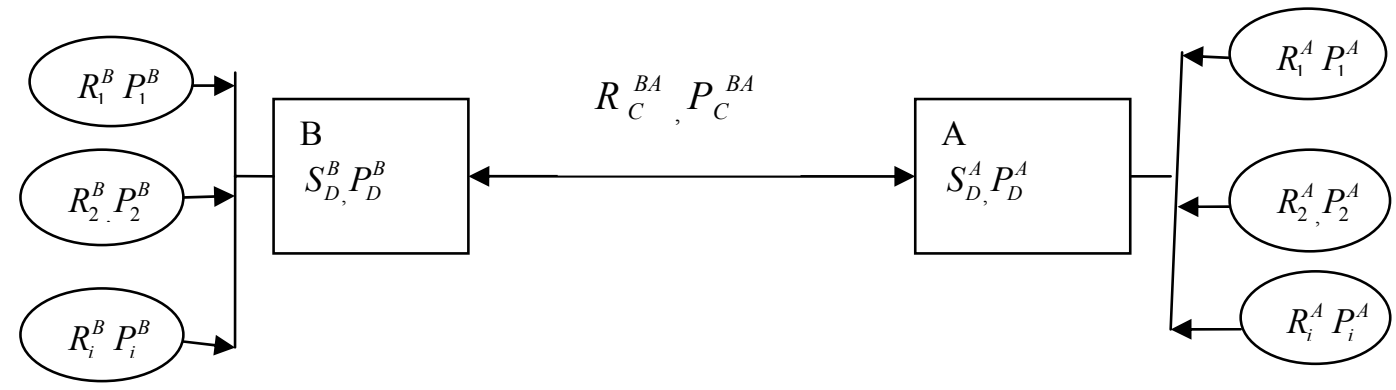

Figure 1. Block diagram of multi-area competitive electricity market

The objective function i.e total market $\operatorname{cost} C_{T}$ as given below should be minimized.

$$
C_{T}=\sum_{i=1}^{k+l}\left[F\left(P_{i}\right)+G\left(R_{i}\right)\right]
$$

The constraints are:

\subsection{Area power balance constraints}

$$
\begin{aligned}
& \sum_{i=1}^{k} P_{i}^{A}+P_{C}^{B A}=P_{D}^{A} \\
& \sum_{i=1}^{l} P_{i}^{B}-P_{C}^{B A}=P_{D}^{B}
\end{aligned}
$$

\subsection{Spinning reserve constraints}

$$
\sum_{i=1}^{K} R_{i}^{A}+R_{C}^{B A}=R_{D}^{A}
$$




$$
\sum_{i=1}^{l} R_{i}^{B}-R_{C}^{B A}=R_{D}^{B}
$$

\subsection{Unit energy and reserve capacity constraints}

$$
\begin{aligned}
& P_{i}^{\min }<P_{i}<P_{i}^{\max } \\
& 0<R_{i}<R_{i}^{\max } \\
& P_{i}+R_{i}<P_{i}^{\max }
\end{aligned}
$$

Spinning reserve is the resource capability which is synchronized to the power system and is fully available in a short time.

\subsection{Transmission capacity constraints}

$-P_{C}^{\max }<P_{C}^{B A}<P_{C}^{\max }$

\subsection{Calculation of total market cost}

In this paper, each generator offers a sectional energy offering price curve and a sectional spinning reserve offering price curve. In both curves of each generator, the output power interval corresponds to an energy offering price and a spinning reserve offering price. Take the output power interval k in Figure 2 as an example, if the output power $P_{G i k}$ is less than or equal to $\overline{P_{G i k}}$ and larger than $\overline{P_{G i(k-1)}}$, the offering prices for the energy and the spinning reserve are $\rho_{G i k}$ and $\rho_{R i k}$, respectively.

In this paper, the energy and reserve bidding prices are calculated as

$F\left(P_{i}\right)=\left\{\begin{array}{l}\rho_{G i(k-1)} \times P_{i} \ldots . . \text { if } \ldots P_{i} \leq P_{G i(k-1)} \\ \rho_{G i(k-1)} \times P_{G i(k-1)}+\left(P_{i}-P_{G i(k-1)}\right) \times \rho_{G i k} \ldots \ldots . . . . . . P_{G i(k-1)}<P_{i} \leq P_{G i k} \\ \rho_{G i(k-1)} \times P_{G i(k-1)}+\left(P_{G i k}-P_{G i(k-1)}\right) \times \rho_{G i k}+\left(P_{i}-P_{G i k}\right) \times \rho_{G i(k+1)} \ldots \text { if } \ldots . . P_{G i k} \leq P_{i}\end{array}\right.$

$G\left(R_{i}\right)=\left\{\begin{array}{l}\rho_{R i(k-1)} \times R_{i} \ldots . i f \ldots R_{i} \leq P_{R i(k-1)} \\ \rho_{R i(k-1)} \times P_{R i(k-1)}+\left(R_{i}-P_{R i(k-1)}\right) \times \rho_{R i k} \ldots \ldots . i f \ldots . P_{R i(k-1)}<R_{i} \leq P_{R i k} \\ \rho_{R i(k-1)} \times P_{R i(k-1)}+\left(P_{R i k}-P_{R i(k-1)}\right) \times \rho_{R i k}+\left(R_{i}-P_{R i k}\right) \times \rho_{R i(k+1)} \ldots \text { if } \ldots P_{R i k} \leq R_{i}\end{array}\right.$

\section{Classical Differential Evolution}

Evolutionary optimization methods have emerged as popular alternate approaches for solving power system optimization and other problems, because these methods are based on natural phenomena and therefore are more robust and suitable for practical problems having a real world flavor. Out of the different evolutionary methods, PSO and DE techniques have emerged as most popular. This section presents an i) in-depth review and comparison of various DE strategies ii) impact of tuning parameters.

$\mathrm{DE}$ is a population-based stochastic optimization method based on evolutionary computation. DE differs from conventional genetic algorithms in its use of perturbing vectors, which are the difference between two randomly chosen parameter vectors. The DE algorithm was first introduced by Storn and Price in 1995 (Storn et al,1995) and was successfully applied in the optimization of some well-known nonlinear, non-differentiable, and non-convex functions. DE works on three basic operations, namely mutation, crossover and selection. 


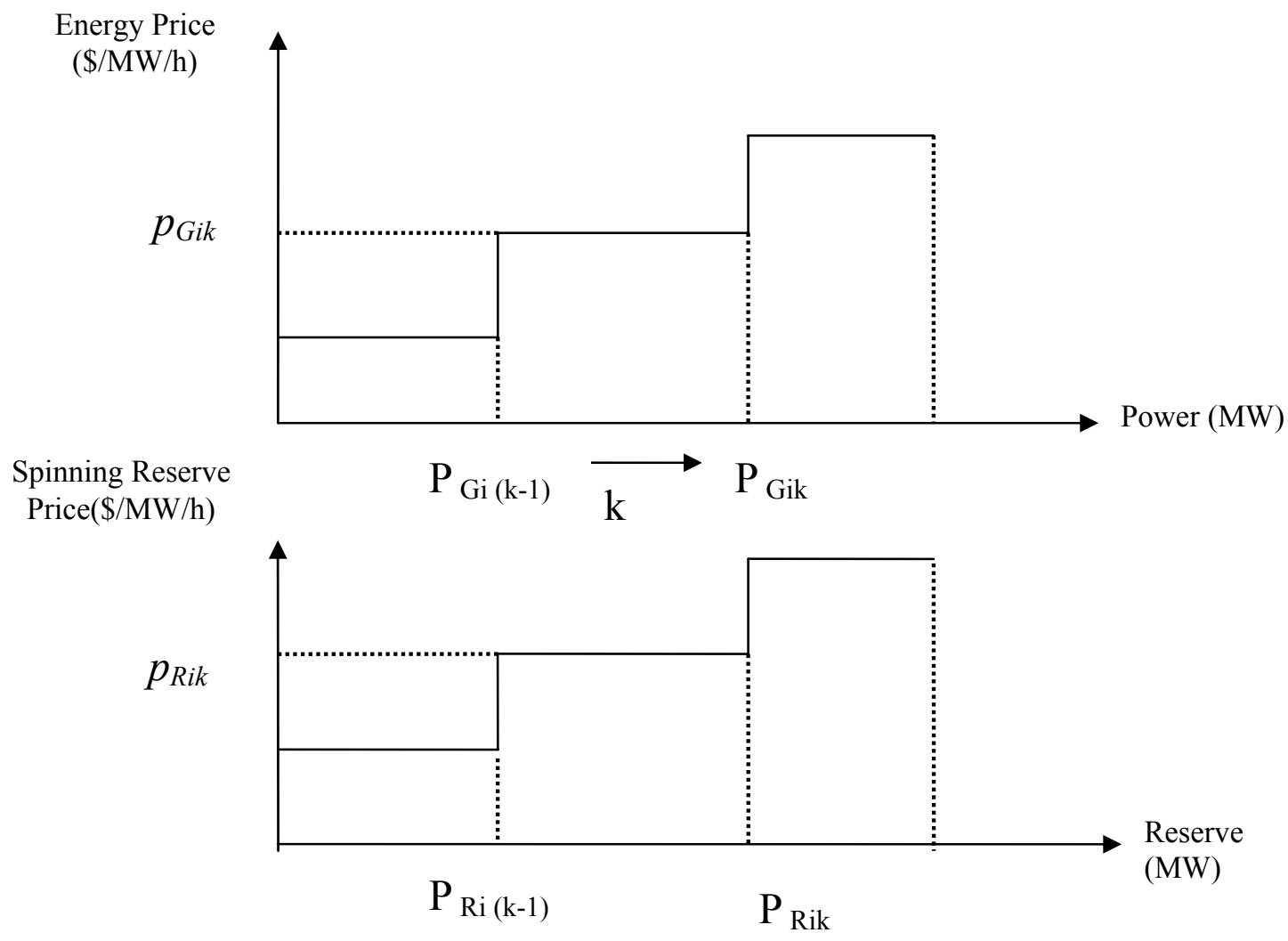

Figure 2. Sectionalized price blocks for energy and reserve

Mutation: This is an operation that adds a vector differential to a population vector of individuals according to the chosen $\mathrm{DE}$ mutation strategy. The different strategies of $\mathrm{DE}$ are classified using the notation $\mathrm{DE}, / \alpha / \beta / \delta$ where $\alpha$ indicates the method for selecting the parent chromosome that will form the base of the mutated vector, $\beta$ indicates the number of difference vectors used to perturb the base chromosome, and $\delta$ indicates the recombination mechanism used to create the offspring population. Much work has not been done on these different strategies; most researchers have explored the variant DE / rand / $1 /$ bin which implies, random selection, one difference vector and binomial crossover. The best performing strategy is found to be problem specific and needs detailed investigation (Sharma et al, 2010). The donor or mutant vector for each population member is generated for different variants in classic DE as given below

1) Mutation Strategy I: DE/rand/1

$$
Z_{i}(t+1)=x_{i, r 1}(t)+f_{m}\left[x_{i, r 2}(t)-x_{i, r 3}(t)\right]
$$

2) Mutation Strategy II: DE/best/1

$$
Z_{i}(t+1)=x_{i, \text { best }}(t)+f_{m}\left[x_{i, r 2}(t)-x_{i, r 3}(t)\right]
$$

3) Mutation Strategy III: DE/rand-to-best/1

$$
Z_{i}(t+1)=x_{i}(t)+f_{m}\left[x_{i, \text { best }}(t)-x_{i}(t)\right]+f m\left[x_{i} r_{1}(t)-x_{i} r_{2}(t)\right]
$$

4) Mutation Strategy IV: DE/best $/ 2$

$$
Z_{i}(t+1)=x_{i_{\text {best }}}(t)+f_{m}\left[x_{i, r 1}(t)-x_{i} r_{2}(t)\right]+f m\left[x_{i} r_{3}(t)-x_{i} r_{4}(t)\right]
$$

5) Mutation Strategy V: DE/rand/2

$$
Z_{i}(t+1)=x_{i r 5}(t)+f_{m}\left[x_{i, r 1}(t)-x_{i} r_{2}(t)\right]+f m\left[x_{i} r_{3}(t)-x_{i} r_{4}(t)\right]
$$

where $i=1,2 \ldots, R$ is the individual's index of population, $t$ is the time (generation); $r_{1}, r_{2}, r_{3}, r_{4}$ and $r_{5}$ and are mutually different integers and also different from the running index,$i$, randomly selected with uniform distribution from the population set and fm $>$ 0 is a real parameter called mutation factor, which controls the amplification of the difference between two individuals so as to avoid search stagnation and is usually taken from the range $[0,2]$. 
Crossover Operation: After the mutation operation, crossover operation is performed to generate a trial vector $U_{i}$ by replacing certain parameters of the target vector $\left(x_{i}\right)$ with the corresponding parameters of the randomly generated donor or mutant vector $\left(Z_{i}\right)$.

$$
U_{i j}(t+1)=\left\{\begin{array}{l}
Z_{i j}(t+1), \ldots . . i f(\operatorname{rand}(j) \leq C R) \operatorname{or}(j=\operatorname{rand} \operatorname{int}(i)) \\
x_{i j}(t), \ldots \ldots \ldots i f(\operatorname{rand}(j)>C R) \operatorname{or}(j \neq \operatorname{rand} \operatorname{int}(i))
\end{array}\right.
$$

In the above, rand(j) is the $\mathrm{j}^{\text {th }}$ evaluation of a uniform random number generation within range $[0,1], C R$ is a crossover rate in the range $[0,1]$. The performance of a $\mathrm{DE}$ algorithm usually depends on three variables; the population size $\mathrm{R}$, the mutation factor $f_{m}$ and the recombination rate $C R$.

Selection: It is the procedure to decide whether the generated trial vector should replace the target vector and be a member of the population in the next generation. In this case, the cost of each trial vector $U_{i}(t+1)$ is compared with that of its parent target vector $x_{i}(t)$ and selection for a minimization problem can be carried out using the following:

$$
x_{i}(t+1)=\left\{\begin{array}{l}
U_{i}(t+1) \ldots . . i f\left(u(t+1)<f\left(x_{i}(t)\right)\right. \\
x_{i}(t), \ldots \text { otherwise }
\end{array}\right.
$$

\subsection{Time- varying differential evolution}

The complex behavior of a non-linear, deterministic, dynamical system which is highly sensitive to initial conditions can be described by Chaos ( $\mathrm{Li}$ et al,1998). Premature convergence to local minima in evolutionary optimization methods can be prevented by employing chaotic sequences in place of random sequences (Li et al,1998, Caponetto et al, 2003, Coelho et al, 2006). Coelho and Mariani (Coelho et al, 2006) integrated chaotic sequences with the mutation factor in differential evolution to improve solution quality. Caponetto (Caponetto et al, 2003) proposed various chaotic sequences in evolutionary algorithms (EAs) in place of the random numbers. .

Mutation rate $f m$ and crossover rate $C R$ in the DE algorithm, significantly affect the performance of the algorithm. The smaller the mutation rate $f m$, longer time will be required for convergence. Larger values of $f m$ allow exploration due to which the algorithm may not converge and skip good optimal solution. The value of $f m$ should be small enough to enable the algorithm to explore tight valleys and large enough to allow global exploration in order to maintain population diversity. A higher $C R$ creates more diversity and better exploration in the new population. In classical DE both $f m$ and $C R$ are fixed, so a lot of parameter tuning is required to achieve global best results. This problem can be solved by employing time-varying mutation and crossover rates. The parameters can be varied with time linearly or in a random manner. The use of chaotic sequences in DE is useful to escape easily from local minima. One of the simplest dynamic systems evidencing chaotic behavior is the iterator called the logistic map (Li et al,1998), whose equation is given by

$y(t)=\mu \times y(t-1) \times[1-y(t-1)]$

where $t$ is the iteration count and $\mu$ is a control parameter, $0 \leq \mu \leq 4$. The behavior of the system represented by eq. (19) significantly changes with the variation in $\mu$. The value of $\mu$ controls the variation of the chaotic sequence. Three DE variants with time-varying $f_{m}$ and $C R$ are proposed here. The variation of $f m$ chaotically, based on logistic map and time varying crossover rate $C R$, give rise to the following time-varying $\mathrm{DE}$ variants:

Variant $1 /$ TVDE(1): The parameter $f_{m}$ is varied as per equation (19) where $f_{m}(0)$ lies between $[0,1]$. The index ' $t$ ' is the current iteration and $f_{m}(t)$ is the new mutation factor based on the logistic map.

$f_{m}(t)=\mu \times f_{m}(t-1) \times\left[1-f_{m}(t-1)\right]$

The parameter $\mu$ decides whether the mutation rate $f_{m}$ oscillates between a limited sequence, varies chaotically or stabilizes to a constant value. A very small difference in $f_{m}(0)$ causes significant difference in its variation pattern. The system at $(20)$ is deterministic and displays chaotic behavior when $\mu=4$ and $f_{m}(0) \notin\{0,0.25,0.5,0.75,1.0\}$.

Variant $2 /$ TVDE(2): The parameter $f m$ is decreased from an initial value $f_{2 i}$ to a final value $f_{2 f}$ with the progress of the optimization algorithm, as per the dynamics given below:

$f_{1}(t)=\mu \times f_{1}(t-1) \times\left[1-f_{1}(t-1)\right]$ 
$f_{m}(t)=\left[\left(f_{2 f}-f_{2 i}\right) \frac{\text { iter }}{\text { iter }_{\max }}+f_{2 i}\right] f_{1}(t)$

Variant 3/TVDE(3): The parameter $f_{m}$ is increased from an initial value $f_{2 i}$ to a final value $f_{2 f}$ with the iterative progress of the optimization algorithm, as per the dynamics given by (21) and (22) by suitable choice of initial and final values of mutation rate. The value of $C R$ in all the above cases is changed iteratively as given below:

$C R($ iter $)=\left(C R_{\max }-C R_{\min }\right) \times \frac{\left(\text { iter }_{\max }-\text { iter }\right)}{\text { iter } r_{\max }}+C R_{\min }$

The variation of $f m$ and $C R$ for $\mu=3$ has been shown for the above three time-varying DE variants in Figure 3(a) -Figure 3(c) respectively.

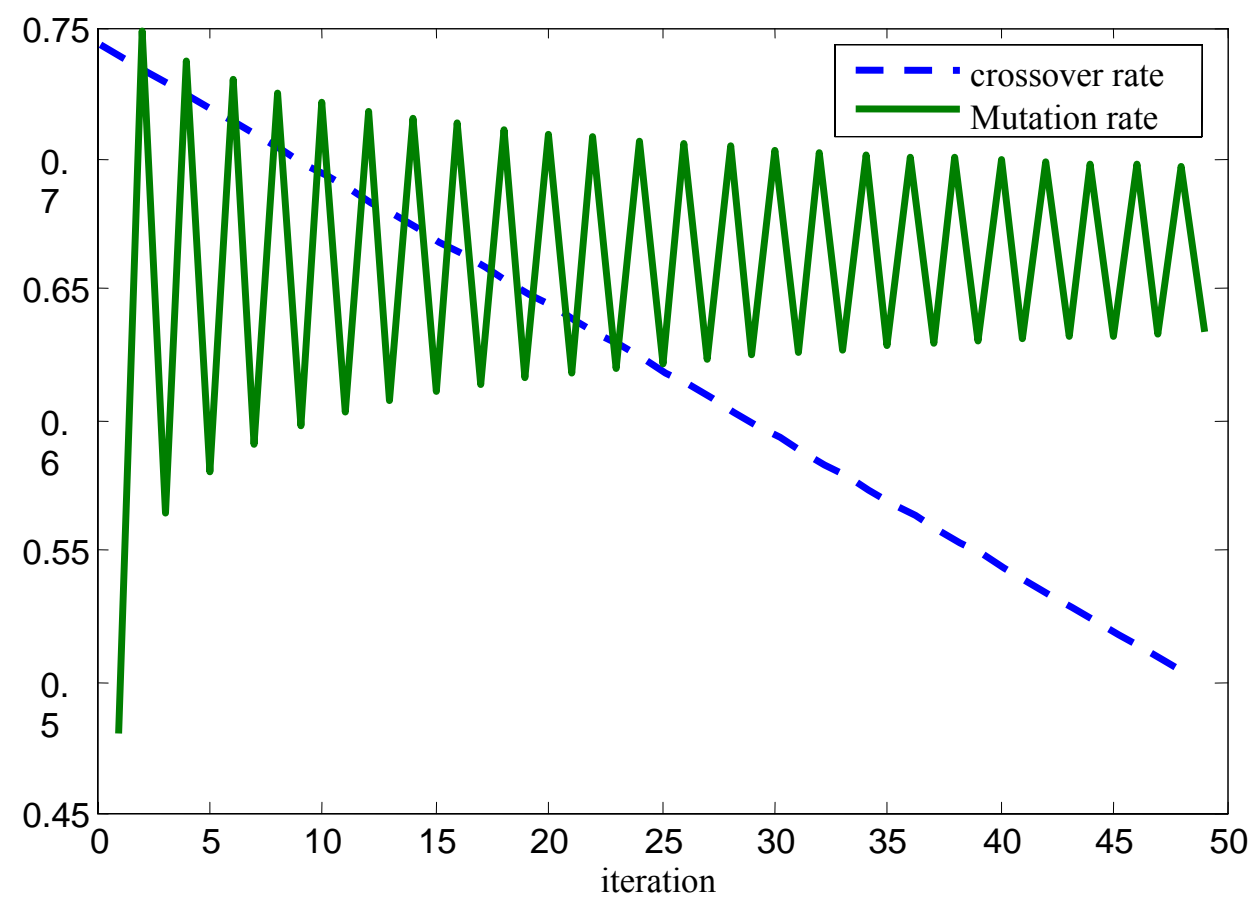

Figure 3(a). Variation of $f_{m}$ for TVDE1 strategy; $\mu=3, f_{l}(t=0)=0.48$ 


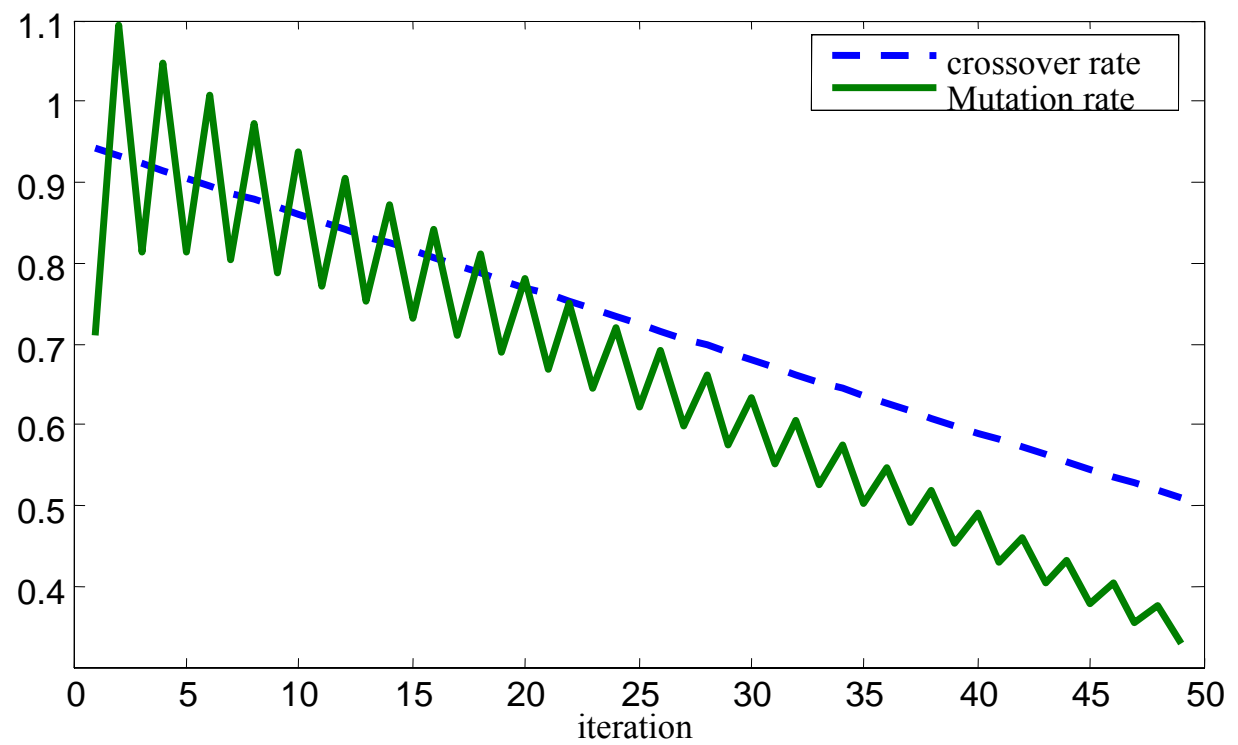

Figure 3(b). Variation of $f_{m}$ for TVDE2 strategy; $\mu=3, f_{1}(t=0)=0.48 ; f_{2 i}=1.5 ; f_{2 f}=0.5$;

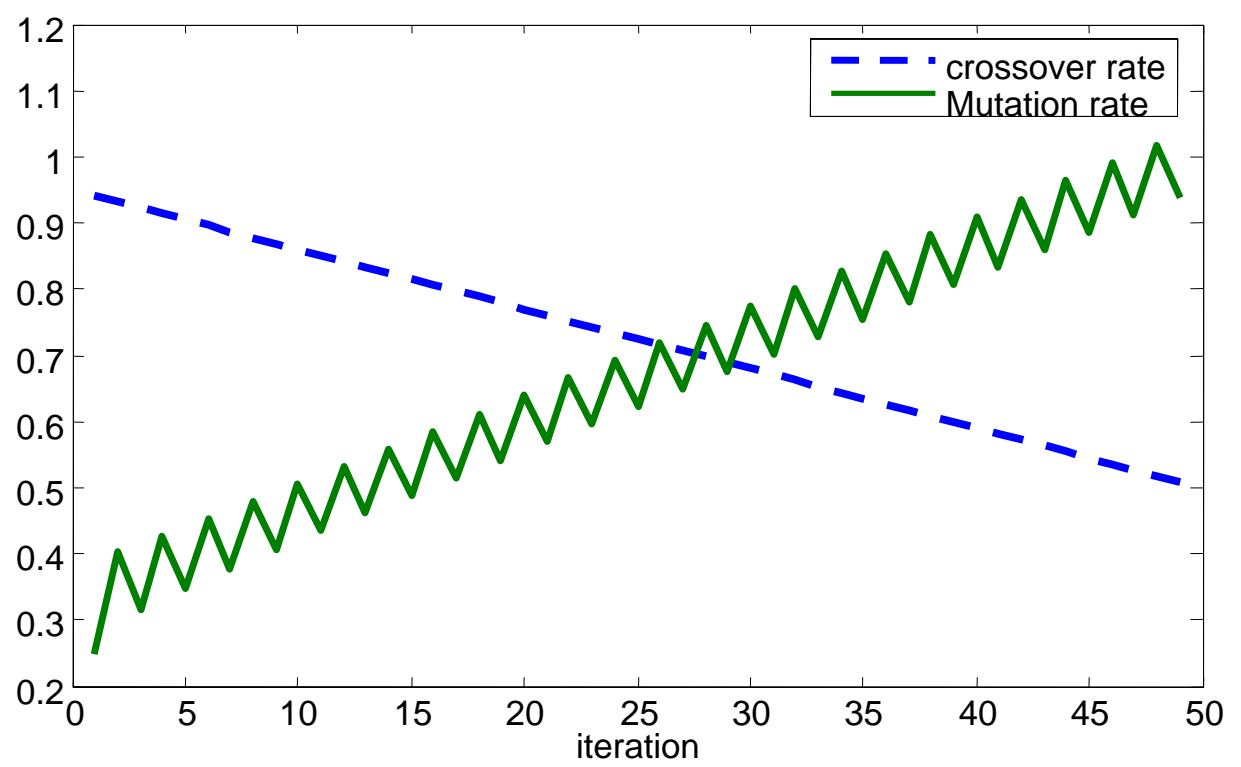

Figure 3(c). Variation of $f_{m}$ for TVDE3 strategy; $\mu=3, f_{l}(t=0)=0.48 ; f_{2 i}=0.5 ; f_{2 f}=1.5$

\section{Solution of JERD using time-varying DE}

This section presents step by step solution of JRED problem employing time-varying differential evolution and critically compares the performance for various DE mutation strategies and proposed variants.

\section{Step 1) Parameter setup}

The DE parameters such as population size, the boundary constraints of optimization variables, mutation strategy to be adopted, the mutation factor $(f m)$, the crossover rate $(C R)$, and the stopping criterion or maximum number of iterations, are selected. 
Step 2) Initialization of an individual population

For fast convergence it is necessary to ensure generation of a feasible initial population vector. The individuals in the population are randomly generated between the maximum and the minimum operating limits of the units respectively such that constraints given by eq.(6)-eq. (8) are satisfied.

The $\mathrm{i}^{\text {th }}$ individual population is represented as

$$
P_{i}=\left(P_{1}, P_{2} P_{3} \ldots \ldots . P_{k+l}, P_{C}^{A B}, R_{1}, R_{2} \ldots \ldots . R_{k+l}\right)
$$

\section{Step 3) Initialization of energy and reserve of the population}

The $\mathrm{j}^{\text {th }}$ dimension (from $\mathrm{j}=1$ to $\mathrm{k}+\mathrm{l}$ ) of the $\mathrm{i}^{\text {th }}$ individual of the population is initialized as given below to satisfy the generation and reserve limit constraints given by eq. (6)-eq. (9). Here, $r_{1}, r_{2}$ and $r_{3}$ are random numbers between [0,1].

$P_{i j}=P_{i j}^{\mathrm{min}}+r_{1}\left(P_{i j}^{\mathrm{max}}-P_{i j}^{\mathrm{min}}\right)$

$R_{i j}=R_{i j}^{\min }+r_{2}\left(R_{i j}^{\max }-R_{i j}^{\min }\right)$

For satisfying constraint given by eq. (8),

$$
\begin{aligned}
& R_{i j}=\min \left\{\left(P_{i j}^{\max }-P_{i j}\right), R_{i j}\right\} \\
& P_{i C}=P_{C}^{\min }+r_{3}\left(P_{C}^{\max }-P_{C}^{\min }\right)
\end{aligned}
$$

Step 4) Fitness evaluation of each individual of the population

The fitness of each individual in the population is evaluated to judge its merit for JERD problem using a function called evaluation function. The evaluation function is defined such that cost is minimized while area wise power balance constraints given by eq. (2)-(3) and reserve balance constraints for all areas given by eq. (4) and eq. (5) respectively are satisfied. The popular penalty function method has proved very effective in modeling complex equality constraints in multi-area and reserve constrained economic dispatch (Sharma et al, 2010). The penalty functions convert a constrained optimization problem into an unconstrained optimization problem. The penalty functions composed of squared or absolute violations are introduced in the fitness function such that an infeasible solution is awarded lesser fitness than the weakest feasible individual string. Since two infeasible individuals are not treated equally, the individual which is further away from the feasibility boundary is more heavily penalized compared to the one which is nearer to the boundary. The evaluation function used here is given by

$$
\min C_{T}+\alpha\left[\sum_{i=1}^{k} P_{i}-P_{C}^{A B}-P_{D}^{A}\right]^{2}+\beta\left[\sum_{i=1}^{l} P_{i}+P_{C}^{A B}-P_{D}^{B}\right]^{2}+\gamma\left[\sum_{i=1}^{k+l} R_{i}-\left(R_{D}^{A}+R_{D}^{B}\right]^{2}\right.
$$

Here, $\alpha, \beta$ and $\gamma$ are the penalty coefficients respectively. The penalty terms impose a penalty on the individual in terms of increased cost, if any of the equality constraints are not satisfied. The first term is calculated for each individual using eq. (1). Large values of penalty coefficients are selected to guarantee feasible solutions and fast convergence.

\section{Step5) Parameter updating using mutation}

The individuals are updated to improve their fitness. In mutation a vector differential is added to the population vector of individuals; the donor vector is generated for the selected DE strategy by using any one equation from eq. (12)-eq. (16).

\section{Step 6) Crossover operation}

Recombination is applied in DE using eq. (17) to generate a trial vector by replacing certain parameters of the target vector with the corresponding parameters of the randomly generated donor in the step 5.

\section{Step 7) Checking the updated population for bound violations}

The upper and lower limits specified by eq. (6) to eq. (9) may get violated after population updating process in step5-step6. Each dimension of an individual is checked for the binding constraints, and made to follow the minimum and maximum limits. Violated values are made to assume the nearest value on feasibility boundary. 
Step 8) Selection operation

The values of the evaluation function are calculated for the updated positions of the individuals. The trial vector $U_{i}(t+1)$ replaces its parent target vector $x_{i}(t)$ if its cost is found to be better otherwise the target vector is allowed to proceed to the next generation.

Step 9) Stopping criterion:

A stochastic optimization algorithm is stopped either based on the tolerance limit or maximum number of iterations. The number of iterations is adopted as the stopping criterion in this chapter.

The detailed flow chart given in Figure 4 details the procedure followed for the JERD problem using TVDE approach.

Select population size, minimum and maximum limits (of energy, reserve, tie-limits, mutation factor, and crossover rate), number of iterations, mutation strategy etc.

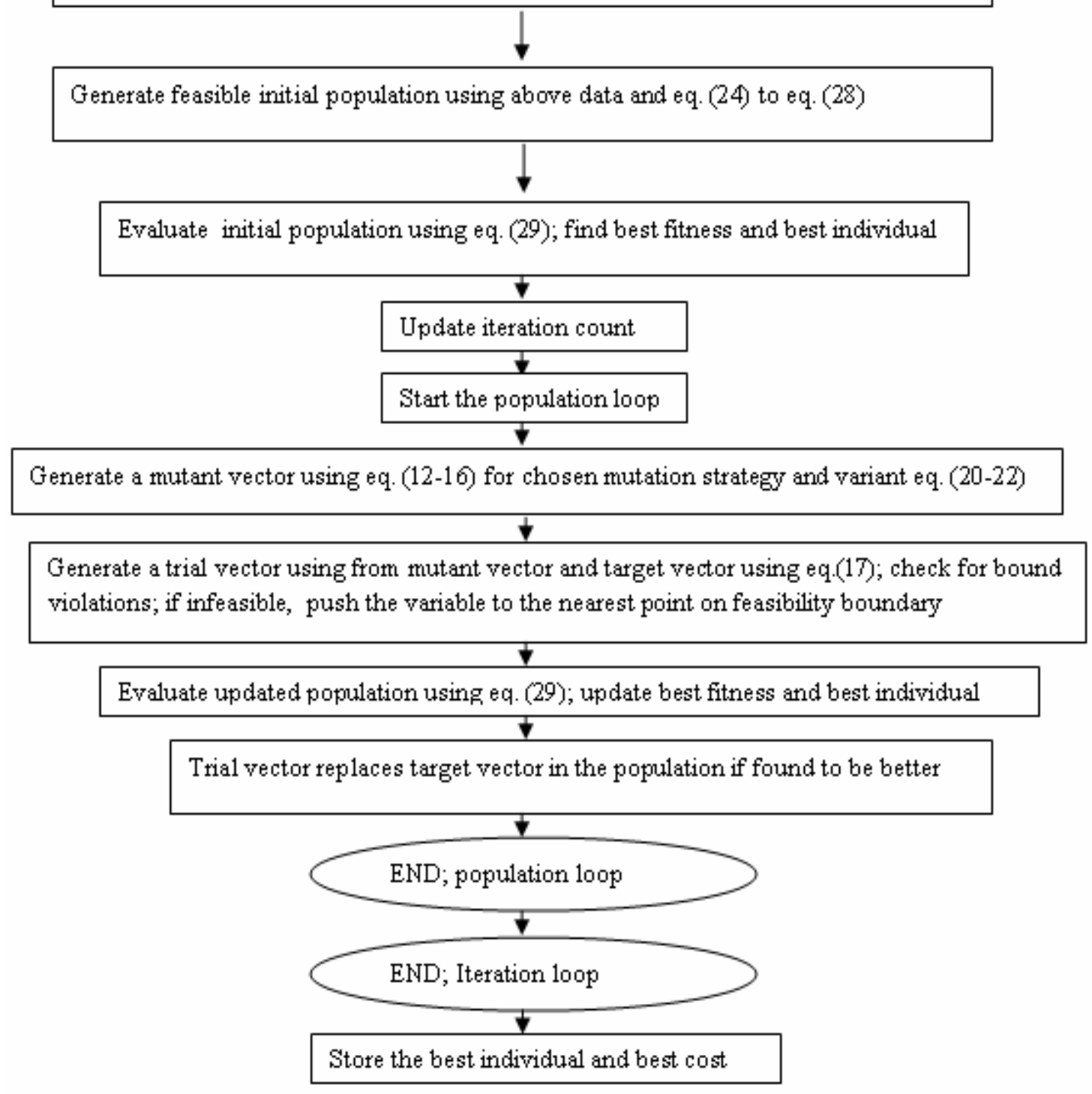

Figure 4. Flow chart of proposed TVDE for JERD problem 


\section{Results and Discussions}

The different DE mutation strategies are tested for the proposed practical JERD problem on two test cases. The first case is New Zealand market data taken from (X. Ma et al, 1998) and multi-area operation is carried out. Complex constraints like reserve requirements, zonal power balance constraints, area spinning reserve constraints, tie-line constraints and reserve and capacity coupling constraints have been included in the joint energy and reserve dispatch (JERD) model defined by equations (1) to (11). Demand elasticity has been considered and objective function defined by eq.(1), i.e .total cost of energy and reserve is optimized using a time-varying DE algorithm. Security constraints have not been formulated in this work, however the security constraints (voltage limits, power flow limits etc.) can also be implemented in a similar manner. The results are in agreement with previously published results (Nasr azdani et al, 2010) and also match with PSO_TVAC technique.

The different DE mutation strategies are tested for the proposed practical JERD problem on two test cases. The performance of different classical DE strategies is compared for time varying DE variants and with PSO_TVAC (Ratnaweera et al, 2004) technique. The obtained results are also compared with previously published results (Azadani et al, 2010). Simulations were carried out using MATLAB 7.0.1 on a Pentium IV processor, 2.8 GHz. with 1 GB RAM.

\subsection{Description of test systems}

Case I: It is a six-generating unit, 2-area system, with three units in each area. The data is given in Table A1 in the appendix (Azadani et al, 2010). Total load demand is $1040 \mathrm{MW}$ which is equally divided between the two areas, reserve requirement is 100 MW. The transmission limit is taken as $240 \mathrm{MW}$.

Case II: In this system, there are four units in area one and two units in area two. The area loads are 113 MW and 84 MW respectively, reserve requirement is $33 \mathrm{MW}$ and tie-line limit is $20 \mathrm{MW}$. The price data is given in Table A2 in the appendix section. The block diagram of this system is given in Figure 5.

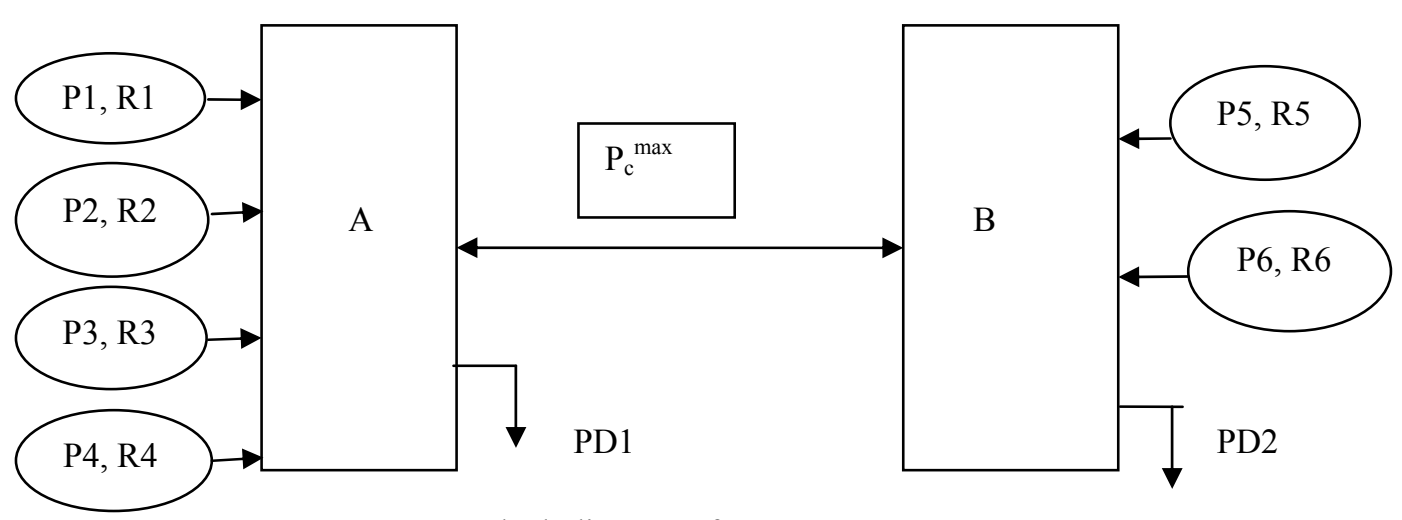

Figure 5. Block diagram of Test Case II

\subsection{Parameter set-up}

Evolutionary techniques employ random operators for updating parameters, as a result of which the results obtained in every fresh trial are slightly different. Therefore, to establish the consistency of these methods, results are compiled out of a number of trials, with different random initial populations. Thirty different trials with a population size of 50 with 1500 number of iterations, are carried out in this paper for both test cases and the obtained mean cost and standard deviation (S.D.) is used to compare the different strategies and variants.

\subsection{Comparison of classical DE mutation strategies}

In the present paper, a detailed comparison is carried out for finding out the best mutation strategy (eq. (12) - eq. (16)) for JERD problem using mean cost and its standard deviation (S.D.) as the performance measures. Both the test cases failed to converge for mutation strategy II and V. To highlight the dependence of classical DE on tuning parameters, the variation of mean cost and S.D. for case II is plotted in Figure 6-Figure 8 for strategy I, strategy III and strategy V respectively, for different values of $C R$ and $f m$. It is clear from these three Figures that classical DE works effectively in a very narrow range of tuning parameters. For example, for strategy I better results are obtained for low and medium values of $f_{m}$ and $C R$ while for strategy III and IV lesser S.D. is 
obtained for medium and larger values of $f_{m}$ and $C R$. This dependence on tuning parameters is eliminated when the TVDE variants proposed in section 3.1 are implemented as shown ahead.

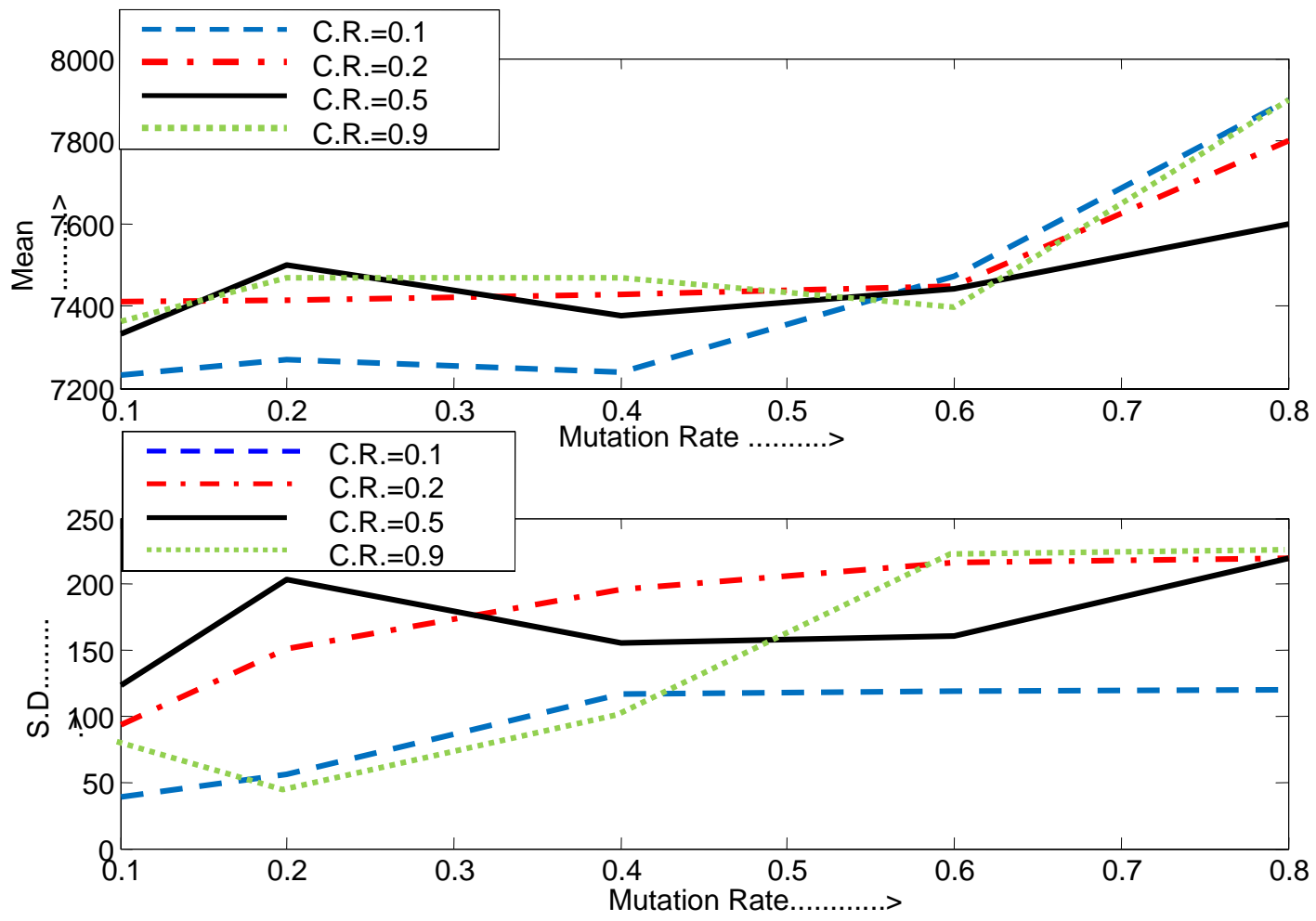

Figure 6. Effect of Mutation rate and C.R. on Mean cost and S.D. for Strategy I
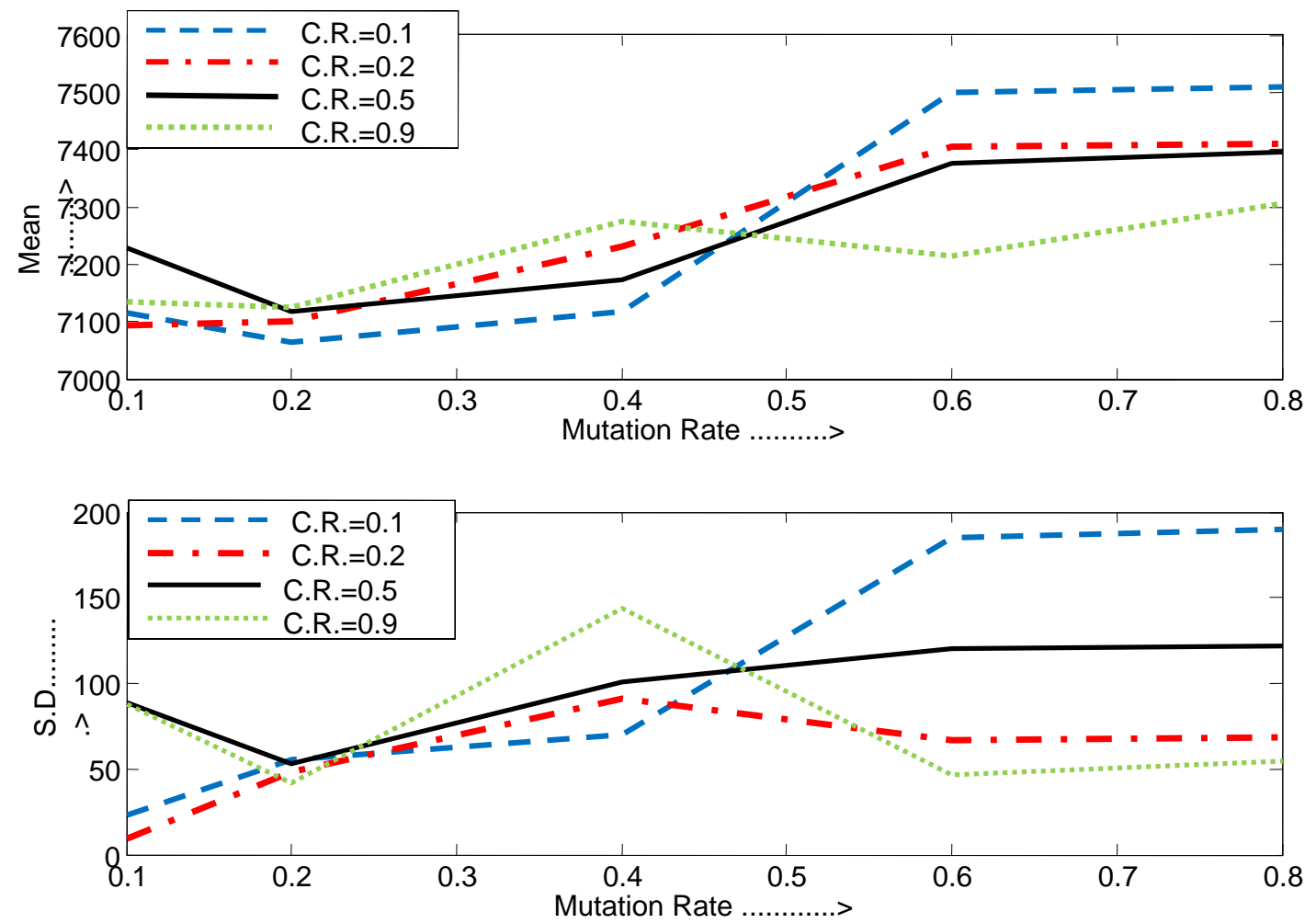

Figure 7. Effect of Mutation rate and C.R. on Mean cost and S.D. for Strategy III 

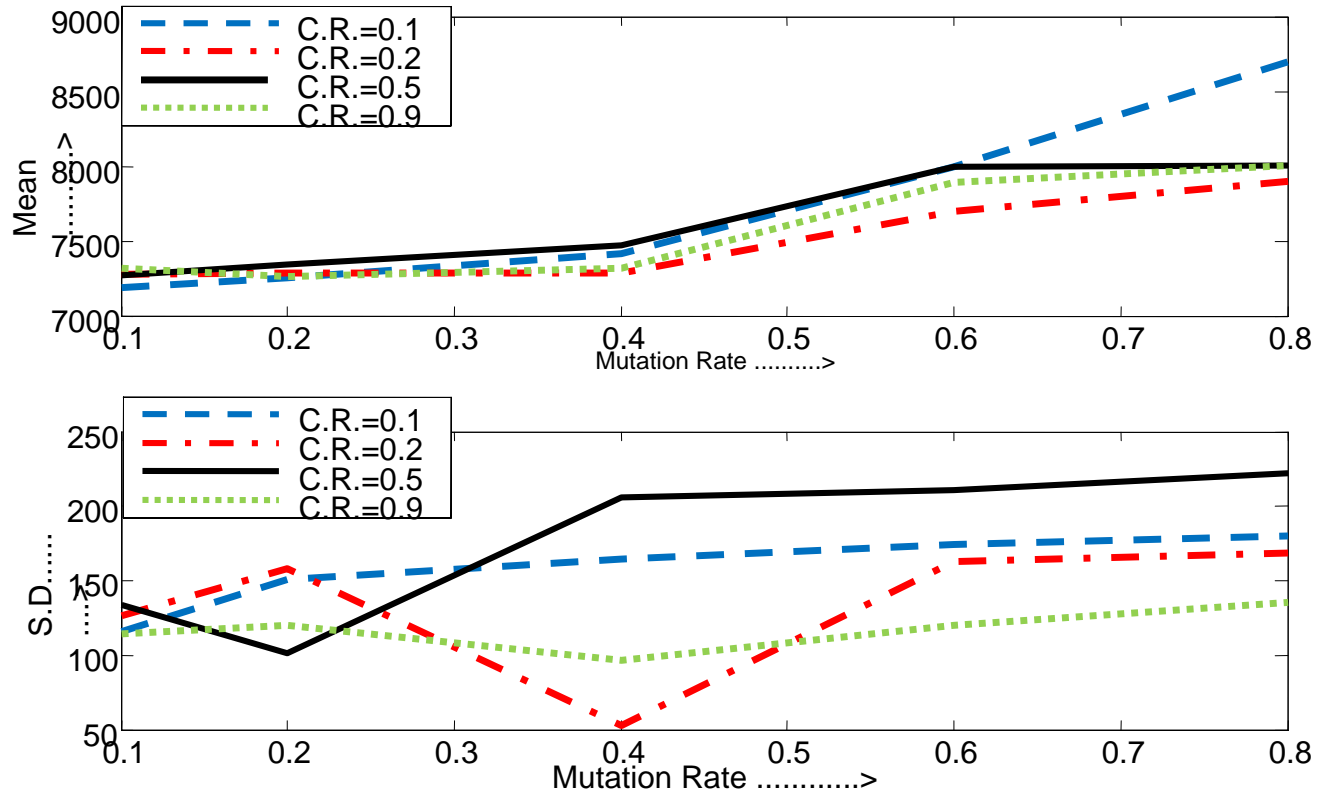

Figure 8. Effect of Mutation rate and C.R. on Mean cost and S.D. for Strategy IV

\subsection{Time varying $D E$ variants}

Table 1 presents the results of TVDE1-TVDE3 for test case I. On comparison with classical DE and PSO_TVAC method the results of TVDE1 and TVDE3 variants are found to be better and closely matching with the results of (Azadani et al, 2010) and PSO_TVAC for Case I. For classical DE strategy III with $f m=C R=0.9$ was adopted. For the case I all techniques converge to the global best value of $\$ 3357.8 / \mathrm{h}$ but classical DE has slight area power balance violations. In Table 2 the results of TVDE1-TVDE3 are compared with PSO_TVAC for Case II It is found that without parameter tuning, classical DE does not converge to the global best value of $\$ 6934.9840 / \mathrm{h}$ for the case II. But all three TVDE methods converged to the best results. As the results of TVDE3 are found to be the best, further results are computed using TVDE3 approach with mutation strategy III and $\mu=3$.

Table 1. Comparison of joint dispatch results for Case I without transmission limits

\begin{tabular}{|c|c|c|c|c|c|c|}
\hline Unit & PSO_TVAC & DE & TVDE1 & TVDE2 & TVDE3 & CPSO[9] \\
\hline A1 & 12.0008 & 11.9973 & 12.0004 & 11.9999 & 12.0009 & 12 \\
\hline A2 & 133.0198 & 133.0238 & 133.0196 & 133.0200 & 133.0191 & 133 \\
\hline A3 & 99.9993 & 99.9987 & 100.0000 & 100.0000 & 100.0000 & 100 \\
\hline B1 & 469.9831 & 469.9800 & 469.9819 & 469.9730 & 469.9807 & 470 \\
\hline B2 & 274.9967 & 274.9984 & 274.9974 & 275.0000 & 274.9993 & 275 \\
\hline B3 & 50.0000 & 50.0015 & 50.0008 & 50.0069 & 50.0000 & 50 \\
\hline Tie-line & -274.9800 & -274.9800 & -274.9800 & -274.9800 & -274.9800 & -275 \\
\hline RA1 & 4.9992 & 5.0000 & 4.9996 & 5.0000 & 4.9991 & 5 \\
\hline RA2 & 20.0000 & 20.0000 & 20.0000 & 20.0000 & 20.0000 & 20 \\
\hline RA3 & 0.0007 & 0.0013 & 0.0000 & 0.0000 & 0.0000 & 00 \\
\hline RB1 & 50.0000 & 50.0000 & 50.0000 & 50.0000 & 50.0000 & 50 \\
\hline RB2 & 5.0033 & 5.0016 & 5.0026 & 5.0000 & 5.0007 & 5 \\
\hline RB3 & 20.0000 & 20.0000 & 20.0000 & 20.0000 & 20.0000 & 20.00 \\
\hline T. M.Res & 100.0032 & 100.0029 & 100.0024 & 100.00 & 100.00 & 100.00 \\
\hline E.M.Cost & 3174.53925 & 3174.5501 & 3174.5389 & 3174.5548 & $\mathbf{3 1 7 4 . 5 3 5 3}$ & 3174.5 \\
\hline R.M.Cost & 183.30340 & 183.30509 & 183.3027 & 183.3000 & $\mathbf{1 8 3 . 2 9 8 4 3}$ & 183.3 \\
\hline T.M.Cost & 3357.8426 & 3357.8552 & 3357.8416 & 3357.8548 & $\mathbf{3 3 5 7 . 8 3 3 7}$ & 3357.80 \\
\hline V1 & -0.0001 & -0.0001 & 0.0000 & -0.0001 & $\mathbf{0 . 0 0 0 0}$ & 0.0000 \\
\hline V2 & -0.0001 & -0.0001 & 0.0000 & -0.0001 & $\mathbf{0 . 0 0 0 0}$ & 0.0000 \\
\hline
\end{tabular}

* V1: Area 'A' power balance violation; V2: Area 'B' power balance violation 
Table 2. Comparison of joint dispatch results for Case II with transmission limit $\left(P_{D}^{A}=113 \mathrm{MW}, P_{D}^{B}=84 \mathrm{MW}\right)$ Tie-line limit=20; Reserve limit=33 MW

\begin{tabular}{|c|c|c|c|c|c|}
\hline Unit & PSO TVAC & DE & TVDE1 & TVDE2 & TVDE3 \\
\hline A1 & 34.9161 & 21.3813 & 33.8446 & 23.3455 & 35.9498 \\
\hline A2 & 30.0808 & 34.4564 & 26.8841 & 35.9992 & 31.9002 \\
\hline A3 & 35.7429 & 36.1238 & 36.2385 & 35.9912 & 24.8283 \\
\hline B1 & 32.2599 & 33.0789 & 30.5118 & 34.5889 & 35.9657 \\
\hline B2 & 35.1093 & 35.3738 & 35.2078 & 35.6447 & 35.1523 \\
\hline B3 & 28.8905 & 36.5857 & 34.3132 & 31.4301 & 33.2038 \\
\hline Tie-line & 20.0000 & 12.0405 & 14.4790 & 16.9250 & 15.6439 \\
\hline RA1 & 5.5000 & 5.5000 & 5.5000 & 5.5000 & 5.5000 \\
\hline RA2 & 5.5000 & 5.5000 & 5.5000 & 5.5000 & 5.5000 \\
\hline RA3 & 5.5000 & 5.5000 & 5.5000 & 5.5000 & 5.5000 \\
\hline RB1 & 5.5000 & 5.5000 & 5.5000 & 5.5000 & 5.5000 \\
\hline RB2 & 5.5000 & 5.5000 & 5.5000 & 5.5000 & 5.5000 \\
\hline RB3 & 5.5000 & 5.5000 & 5.5000 & 5.5000 & 5.5000 \\
\hline Reserve & 33.00 & 33.00 & 33.00 & 33.00 & 33.00 \\
\hline E.M.Cost & 6439.98400 & 6447.0933 & 6442.3818 & 6439.98400 & $\mathbf{6 4 4 0 . 0 0 0 0}$ \\
\hline R.M.Cost & 495.00000 & 495.0000 & 495.0000 & 495.00000 & $\mathbf{4 9 5 . 0 0 0 0}$ \\
\hline T.M.Cost & 6934.98400 & 6942.0933 & 6937.3818 & 6934.9840 & $\mathbf{6 9 3 5 . 0 0 0 0}$ \\
\hline V1 & -0.0002 & 0.0000 & 0.0000 & -0.0002 & $\mathbf{0 . 0 0 0 0}$ \\
\hline V2 & -0.0002 & 0.0000 & 0.0000 & -0.0002 & $\mathbf{0 . 0 0 0 0}$ \\
\hline
\end{tabular}

V1: Area 'A' power balance violation; V2: Area 'B' power balance violation

\subsection{Effect of transmission limits}

Transmission line constraints play an important role in deciding the market cost under a given demand. Table 3 and Table 4 give the joint dispatch results for case I and case II respectively for different tie-line limits for the TVDE3 approach. When the tieline limits are increased from $250 \mathrm{MW}$ to $270 \mathrm{MW}$ the total market cost is reduced from $\$ 3424.3993 / \mathrm{h}$ to $\$ 3364.3297 / \mathrm{h}$ for case I. Similarly, when tie-limits are increased form $5 \mathrm{MW}$ to $20 \mathrm{MW}$ the cost reduced from $\$ 9105.0000 / \mathrm{h}$ to $\$ 9045.0000 / \mathrm{h}$ for $P_{D}^{A}$ $=133 \mathrm{Mw}$ and $P_{D}^{B}=110 \mathrm{MW}$.

Table 3. Effect of transmission limits on joint dispatch results for case I (Reserve $\left.=100 \mathrm{MW} ; P_{D}^{A}=520 \mathrm{MW}, P_{D}^{B}=520 \mathrm{MW}\right)$

\begin{tabular}{|c|c|c|c|}
\hline Generating Units & Tie line limit $=250 \mathrm{MW}$ & Tie line limit $=260 \mathrm{MW}$ & Tie line limit $=270 \mathrm{MW}$ \\
\hline $\mathrm{A} 1(\mathrm{MW})$ & 17.0000 & 17.0000 & 12.4042 \\
\hline A2(MW) & 153.0000 & 143.0000 & 137.7235 \\
\hline A3(MW) & 100.0000 & 100.0000 & 99.8725 \\
\hline B1(MW) & 470.0000 & 470.0000 & 469.5204 \\
\hline B2(MW) & 250.0000 & 260.0000 & 270.2863 \\
\hline B3(MW) & 50.000 & 50.0000 & 50.1931 \\
\hline RA1(MW) & 0.0000 & 0.0000 & 0.3023 \\
\hline RA2(MW) & 20.0000 & 20.0000 & 20.0000 \\
\hline RA3(MW) & 0.0000 & 0.0000 & 0.0012 \\
\hline RB1(MW) & 50.0000 & 50.0000 & 50.0000 \\
\hline RB2(MW) & 30.0000 & 20.0000 & 9.7137 \\
\hline RB3(MW) & 0.0000 & 10.0000 & 19.9828 \\
\hline$P_{C}^{B A}$ (Tie-line flow) MW & -250.00 & -260.00 & -269.9998 \\
\hline Energy Market Cost $(\$ / \mathrm{h})$ & 3280.4993 & 3230.4993 & 3190.4460 \\
\hline Reserve Market Cost $(\$ / \mathrm{h})$ & 143.9000 & 158.6000 & 173.8806 \\
\hline Total Market $\operatorname{cost}(\$ / \mathrm{h})$ & 3424.3993 & 3389.0993 & 3364.3266 \\
\hline Area 'A' Violations (V1) & -0.0000 & -0.0000 & -0.0000 \\
\hline Area 'B' Violations(V2) & -0.0000 & -0.0000 & -0.0000 \\
\hline
\end{tabular}


Table 4. Effect of transmission limits on joint dispatch results for case II ( $P_{D}^{A}=133 \mathrm{MW} ; P_{D}^{B}=110 \mathrm{MW}$; Reserve=33MW)

\begin{tabular}{|c|c|c|c|}
\hline Generating Units & Tie line limit $=20 \mathrm{MW}$ & Tie line limit $=10 \mathrm{MW}$ & Tie line limit $=5 \mathrm{MW}$ \\
\hline A1(MW) & 36.0035 & 35.0699 & 35.4941 \\
\hline A2(MW) & 44.0090 & 35.9585 & 35.9909 \\
\hline A3(MW) & 36.0072 & 35.9927 & 30.5595 \\
\hline B1(MW) & 36.9017 & 35.9789 & 35.9555 \\
\hline B2(MW) & 46.8090 & 52.1416 & 51.6146 \\
\hline B3(MW) & 43.2696 & 47.8584 & 53.3854 \\
\hline RA1(MW) & 5.5000 & 5.5000 & 5.5000 \\
\hline RA2(MW) & 5.5000 & 5.5000 & 5.5000 \\
\hline RA3(MW) & 5.5000 & 5.5000 & 5.5000 \\
\hline RB1(MW) & 5.5000 & 5.5000 & 5.5000 \\
\hline RB2(MW) & 5.5000 & 5.5000 & 5.5000 \\
\hline RB3(MW) & 5.5000 & 5.5000 & 5.5000 \\
\hline$P_{C}^{A B}$ (Tie-line flow) MW & 19.9214 & 10.00 & 5.00 \\
\hline Energy Market Cost $(\$ / \mathrm{h})$ & 8550.0000 & 8560.0000 & 8610.0000 \\
\hline Reserve Market $\operatorname{Cost}(\$ / h)$ & 495.0000 & 495.0000 & 495.0000 \\
\hline Total Market $\operatorname{cost}(\$ / \mathrm{h})$ & 9045.0000 & 9055.0000 & 9105.0000 \\
\hline Area 'A' Violations (V1) & 0.0000 & 0.0000 & 0.0000 \\
\hline Area 'B' Violations(V2) & 0.0000 & 0.0000 & 0.0000 \\
\hline
\end{tabular}

\subsection{Effect of load variation and reserve limits}

The performance of time varying DE variants was also tested for load and reserve requirement variation. The results for the two cases are tabulated in Table 5 and Table 6 for the TVDE3 approach which performed the best out of the three variants.

Table 5. Effect of load variation and reserve limits on joint market cost for case I

\begin{tabular}{|l|l|l|l|c|c|}
\hline \multicolumn{2}{|c|}{$\begin{array}{c}\text { Load Demand } \\
\text { Area1 }\end{array}$} & Reserve(MW) & \multicolumn{1}{c|}{$\begin{array}{c}\text { Energy cost } \\
(\$ / \mathrm{h})\end{array}$} & Reserve cost $(\$ / \mathrm{h})$ & $\begin{array}{c}\text { Total cost } \\
(\$ / \mathrm{h})\end{array}$ \\
\hline \multirow{3}{*}{$520 \mathrm{MW}$} & $520 \mathrm{MW}$ & 100 & 3174.4940 & 183.2997 & 3357.7937 \\
\cline { 3 - 6 } & & 120 & 3247.4926 & 209.9000 & 3457.3926 \\
\cline { 3 - 6 } & & 130 & 3287.4984 & 223.2000 & 3510.6984 \\
\cline { 3 - 6 } & \multirow{3}{*}{$550 \mathrm{MW}$} & 140 & 3325.4983 & 241.8499 & 3567.3482 \\
\cline { 3 - 6 } & \multirow{3}{*}{$550 \mathrm{MW}$} & 80 & 4755.4984 & 148.8000 & 4904.2984 \\
\cline { 3 - 6 } & & 100 & 4785.4984 & 160.0000 & 4945.4984 \\
\cline { 3 - 6 } & & 120 & 4913.4981 & 209.9000 & 4993.7983 \\
\hline
\end{tabular}

Table 6. Effect of load variation and reserve limits on joint market cost for case II

\begin{tabular}{|c|c|c|c|c|c|}
\hline \multicolumn{2}{|c|}{ Load Demand } & \multirow[t]{2}{*}{ Reserve (MW) } & \multirow{2}{*}{$\begin{array}{l}\text { Energy cost } \\
(\$ / \mathrm{h})\end{array}$} & \multirow[t]{2}{*}{ Reserve $\operatorname{cost}(\$ / h)$} & \multirow{2}{*}{$\begin{array}{c}\text { Total cost } \\
(\$ / \mathrm{h})\end{array}$} \\
\hline & Area2 & & & & \\
\hline \multirow[t]{4}{*}{$113 \mathrm{MW}$} & \multirow[t]{4}{*}{$84 \mathrm{MW}$} & 20 & 6439.9997 & 300.0000 & 6739.9997 \\
\hline & & 25 & 6439.9997 & 375.0000 & 6814.9997 \\
\hline & & 30 & 6439.9997 & 450.0000 & 6889.9997 \\
\hline & & 33 & 6439.9997 & 495.0000 & 6934.9997 \\
\hline \multirow{4}{*}{$133 \mathrm{MW}$} & \multirow{4}{*}{$110 \mathrm{MW}$} & 20 & 8549.9995 & 300.0000 & 8849.9995 \\
\hline & & 25 & 8549.9995 & 375.0000 & 8924.9995 \\
\hline & & 30 & 8549.9995 & 450.0000 & 8999.9995 \\
\hline & & 33 & 8549.9995 & 495.0000 & 9044.9995 \\
\hline
\end{tabular}




\subsection{Effect of population size}

To study the effect of population size on time varying DE variants, a statistical analysis was carried out for 30 trials with different population sizes. The results are given in Table 7 for TVDE3. With increase in population size the minimum, maximum, mean cost and S.D. improve as a rule but the computational time increases. It can be seen that the minimum result with zero S.D. was achieved for a population size 50. Therefore the optimal population size was selected at 50 .

Table 7: Effect of population size on performance of TVDE3 for Case II out of 30 trials

\begin{tabular}{|c|c|c|c|c|c|}
\hline Population Size & Minimum & Maximum & Mean & $\begin{array}{c}\text { Standard } \\
\text { deviation }\end{array}$ & CPU time/trial(seconds) \\
\hline 10 & 7108.6700 & 15906.0 & 13450.9 & 357.68 & 1.062 \\
\hline 20 & 6937.6711 & 7500.7 & 7154.8 & 80.55 & 1.812 \\
\hline 50 & 6934.9840 & 6934.9840 & 6934.9840 & 0.0 & 3.89 \\
\hline 100 & 6934.9840 & 6934.9840 & 6934.9840 & 0.0 & 7.391 \\
\hline
\end{tabular}

\subsection{Convergence characteristics and consistency analysis}

The time varying DE variants were found to have a stable convergence characteristic for all tested cases, under all possible variations of demand, reserve, tie-line limit and population size. On comparison with classical DE, the convergence behavior of TVDE schemes was found to be better. Figure 9 shows that out of the three TVDE variants the performance of TVDE3 was found to be the best. Figure 10 compares the best results of 30 trials of the DE variants and it can be seen that TVDE3 is the most consistent approach.

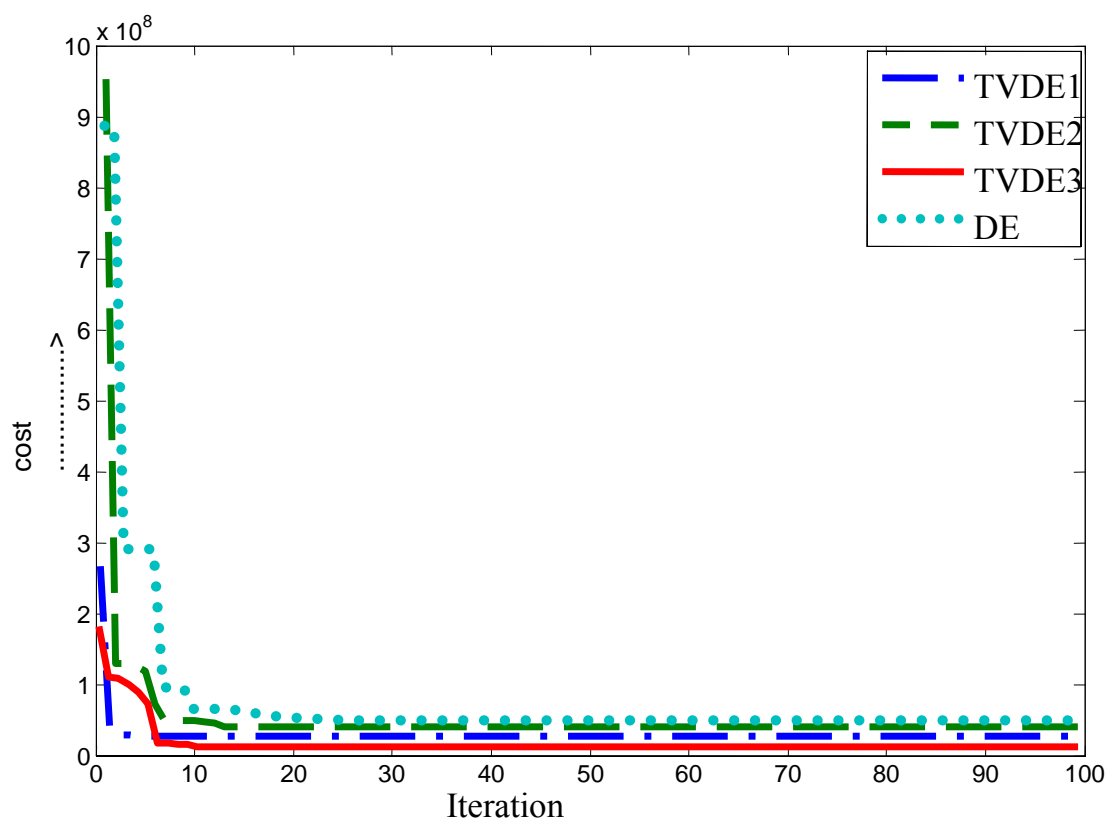

Figure 9. Comparison of convergence characteristics of DE variants for Case I 


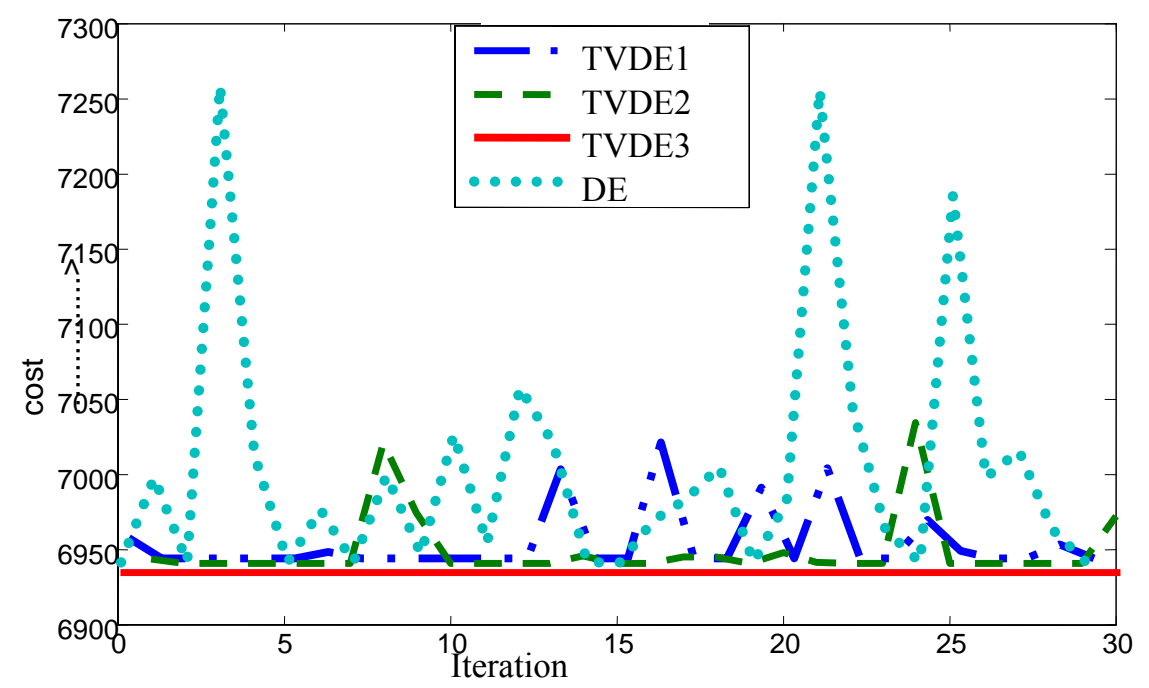

Figure 10 Comparison of best performance of DE variants for case II

\section{Conclusions}

The multi-area joint energy and reserve dispatch problem for the competitive market is more complex as compared to the conventional economic dispatch due to the individual area power balance constraints, reserve requirement constraints, reserve and energy mutual dependence constraints and tie-line constrains. Evolutionary optimization methods are becoming very popular for solving such complex constrained optimization problems due to their population based excellent parallel search capability and superior constraint handling mechanism. To avoid the cumbersome process of parameter tuning in classical DE, a time varying differential evolution approach is proposed in this paper for solving multi-area JERD problem. The major findings can be summarized as:

- A detailed study of DE mutation strategies was carried out and strategy III i.e. DE/rand to best/1 was found to produce the best results. Strategy II and V did not converge for the tested cases.

- The solution quality of classical DE was found to be highly dependant on the values of mutation and crossover rates. The best DE performance is achieved for only certain combinations of tuning parameters $f_{m}$ and $C R$; for other values the results were quite inferior. This limitation of classical DE was removed in the time varying DE variants proposed in this paper. The performance of TVDE variants was found to be superior to classical DE.

- The performance of TVDE was tested with change in load demand, reserve requirements, tie-line limits and population size. In all cases TVDE was found to converge to the global best solution with a small S.D. While all the TVDE schemes performed efficiently, the performance of TVDE3 was found to be the best for the tested JERD cases. The best strategies as well as the best variant are problem dependant as their performance depends on the nature of the optimization function and constraints. For any one type of optimization function same variant performs the best while for a different problem, another variant may work better.

Appendix

Table A1. Market Price Data for Test case I

\begin{tabular}{|c|c|c|c|c|c|c|c|c|c|}
\hline \multirow{3}{*}{ Unit } & \multicolumn{6}{|c|}{ Energy offer } & \multicolumn{2}{|c|}{ Reserve offer } & \multirow{3}{*}{$\begin{array}{l}\text { MW } \\
\text { limit }\end{array}$} \\
\hline & \multicolumn{2}{|c|}{ Band 1} & \multicolumn{2}{|c|}{ Band 2} & \multicolumn{2}{|c|}{ Band 3} & \multicolumn{2}{|c|}{ Band 1} & \\
\hline & MW & Price & MW & Price & MW & Price & MW & Price & \\
\hline $\mathrm{A} 1$ & 5 & -2.3 & 7 & 23 & 5 & 27 & 5 & 3.33 & 17 \\
\hline $\mathrm{A} 2$ & 80 & -2.6 & 60 & 26 & 60 & 28 & 20 & 2.40 & 200 \\
\hline A3 & 70 & -2.0 & 15 & 22 & 15 & 24 & 10 & 2.40 & 100 \\
\hline $\mathrm{B} 1$ & 400 & -2.4 & 60 & 21 & 60 & 24 & 50 & 1.12 & 520 \\
\hline $\mathrm{B} 2$ & 200 & -3.0 & 40 & 17 & 40 & 23 & 50 & 1.33 & 280 \\
\hline B3 & 50 & -2.4 & 30 & 27 & 30 & 29 & 20 & 2.80 & 110 \\
\hline
\end{tabular}


Table A2. Market Price Data for Test case II

\begin{tabular}{|c|c|c|c|c|c|c|c|c|c|}
\hline \multirow{3}{*}{ Unit } & \multicolumn{6}{|c|}{ Energy offer } & \multirow{2}{*}{\multicolumn{2}{|c|}{$\begin{array}{c}\text { Reserve offer } \\
\text { Band } 1\end{array}$}} & \multirow{3}{*}{$\begin{array}{l}\text { MW } \\
\text { limit }\end{array}$} \\
\hline & \multicolumn{2}{|c|}{ Band 1} & \multicolumn{2}{|c|}{ Band 2} & \multicolumn{2}{|c|}{ Band 3} & & & \\
\hline & MW & Price & MW & Price & MW & Price & MW & Price & \\
\hline A1 & 12 & 20 & 24 & 40 & 24 & 50 & 5.5 & 15 & 60 \\
\hline A2 & 12 & 20 & 24 & 40 & 24 & 50 & 5.5 & 15 & 60 \\
\hline $\mathrm{A} 3$ & 12 & 20 & 24 & 40 & 24 & 50 & 5.5 & 15 & 60 \\
\hline A4 & 12 & 20 & 24 & 40 & 24 & 50 & 5.5 & 15 & 60 \\
\hline B1 & 12 & 20 & 24 & 40 & 24 & 50 & 5.5 & 15 & 60 \\
\hline B2 & 12 & 20 & 24 & 40 & 24 & 50 & 5.5 & 15 & 60 \\
\hline
\end{tabular}

\section{Acknowledgement}

The authors sincerely acknowledge the financial support provided by UGC under major research project entitled Power System Optimization and Security Assessment Using Soft Computing Techniques, vide F No.34-399/2008 (SR) dated, $24^{\text {th }}$ December 2008. The authors also thank the Director, M.I.T.S. Gwalior for providing facilities for carrying out this work. The second author acknowledges UGC research award for post doctoral work sanctioned by UGC, New Delhi vide letter no. F-30-120(SC)/2009 (SA-II).

\section{References}

Amjady N., Aghaei J. and Shayanfar H. A. 2009. Stochastic Multiobjective Market Clearing of Joint Energy and Reserves Auctions Ensuring Power System Security, IEEE Transactions on Power Systems, Vol. 24, No. 4, pp. 1841-1854.

Azadani E. N., Hosseinian S.H. and Moradzadeh B., 2010. Generation and reserve dispatch in a competitive market using constrained particle swarm optimization. Electrical Power and Energy Systems, vol. 32, pp. 79-86.

Bai J., Gooi H. B., Xia L. M., Strbac G. and Venkatesh B. 2006.A Probabilistic Reserve Market Incorporating Interruptible Load, IEEE Transactions on Power Systems., Vol. 21, No.3, pp. 1079-1087.

Bakirtzis A.G., 1998. Joint energy and reserve dispatch in a competitive pool using Lagrangian relaxation. IEEE Power Engineering Review, vol. 18, No. 1,pp. 60-62.

Bouffard F. and Galiana F. D.2004. An Electricity Market With a Probabilistic Spinning Reserve, IEEE Transactions on Power Systems, Vol. 19, No. 1, pp. 300-307.

Caponetto R., Fortuna L., Fazzino S., and Xibilia M. G., 2003. Chaotic sequences to improve the performance of evolutionary algorithms. IEEE Trans. Evol. Computation, vol. 7, no. 3, pp. 289-304.

Chaturvedi K. T., Pandit M. and Srivastava L., 2009. Particle Swarm Optimization with Time Varying Acceleration Coefficients for Nonconvex Economic Power Dispatch. Electrical Power and Energy systems, vol. 31, No.6, pp. 249-257.

Chen C. L., 2005. Optimal generation and reserve dispatch in a multi-area competitive market using a hybrid direct search method. Energy Conversion and Management, vol. 46, No. 18-19, pp. 2856-2872.

Cheung K.W, Shamsollahi P., Asteriadis S., Milligan J., and Potishnak M., 1999. Functional requirements of energy and ancillary service dispatch for the interim ISO New England electricity market. IEEE Power Engineering Society Winter Meeting, vol. 1, pp. $269-273$.

Chiou J. P., 2009. A variable scaling hybrid differential evolution for solving large-scale power dispatch problems. IET Generation Transmission Distribution, vol. 3, no. 2, pp. 154-163.

Coelho L. D S. and Mariani V.C., 2006. Combining of chaotic differential evolution and quadratic programming for economic dispatch optimization with valve-point effect. IEEE Trans.on Power System, vol. 21, no. 2, pp. 989-996.

Coelho L. D. S., Souza R. C. T. and Mariani V. C., 2009. Improved differential evolution approach based on cultural algorithm and diversity measure applied to solve economic dispatch problems. Mathematics and Computers in Simulation, vol. 79, pp. 3136-3147.

Deventer A. V., Chowdhury S. , Chowdhury S.P., Gaunt C.T., 2010. Management of emergency reserves dispatch in electricity networks. 2010 International Conference on Power System Technology: Technological Innovations Making Power Grid Smarter, POWERCON2010.

Flynn M., Sheridan W. P., Dillon J. D.and O’Malley M. J. 2001.Reliability and Reserve in Competitive Electricity Market Scheduling, IEEE Transactions on Power Systems., Vol. 16, No. 1, pp. 78 - 87.

Hazrati N., Nejad M. R., and Gharaveisi A.A., 2007. Pricing and Allocation of Spinning Reserve and Energy in Restructured Power Systems via Memetic Algorithm. Large Engineering Systems Conference on Power Engineering, pp. 234 - 238.

Li B. and Jiang W., 1998. Optimizing complex functions by chaos search. Cybern. Syst., vol. 29, no. 4, pp. 409-419. 
Ma X., Sun D. and Cheung K., 1999. Energy and Reserve Dispatch in a Multi-Zone Electricity Market. IEEE Transactions on Power Systems, vol. 14, No. 3, pp. 913-919.

Ma X, and Sun D., 1998. Energy and ancillary service dispatch in a competitive pool. IEEE Power Eng Review, vol 18, No.1, pp 54-56.

Paqaleh M. A. and Hosseini S.H., 2009. Transmission constrained energy and reserve dispatch by harmony search algorithm. IEEE Power \& Energy Society General Meeting , pp. 1 - 8.

Rashidinejad M, Song Y.H. and Dasht-Bayaz M. H. J., 2002. Contingency reserve pricing via a joint energy and reserve dispatching approach. Energy Conversion and Management, vol. 43, pp. 537-548.

Ratnaweera A., Halgamuge S.K. and Watson H.C., 2004. Self-organizing hierarchical Particle swarm optimizer with time varying acceleration coefficients. IEEE Transactions on Evolutionary Computation, vol. 8, No. 3, pp. 240-255.

Shahidehpour M. and Alomoush M., 2001. Restructured Electrical Power Systems, operation, trading and volatility. Marce Dekker Inc.

Sharma M. and Pandit M., 2010. Reserve Constrained Multi-Area Economic Dispatch Employing Evolutionary Approach. International Journal of applied evolutionary computation, vol. 1, No. 3, pp.49-69.

Sharma M., Pandit M. and Srivastava L., 2010. Multi-Area Economic Dispatch with Tie-Line Constraints Employing Evolutionary Approach. International Journal of Engineering Science and Technology (IJEST), (Special Issue on Application of Computational Intelligence in Emerging Power Systems), Vol. 2, No. 3, pp. 133-150.

Storn R. and Price K., 1995. Differential evolution: A simple and efficient adaptive scheme for global optimization over continuous spaces. International Computer Science Institute, Berkeley, CA, Tech. Rep. TR-95-012.

Wen F. and David A.K., 2002. Coordination of bidding strategies in day-ahead energy and spinning reserve markets. International Journal of Electrical Power and Energy Systems, vol. 24, No. 4, pp. 251-261.

Xu Z., Dong Z.Y. and Wong K.P., 2003. Optimal Dispatch of Spinning Reserve in an Competitive Electricity Market using Genetic Algorithms. The 2003 Congress on Evolutionary Computation, Vol. 1, pp. 587-592.

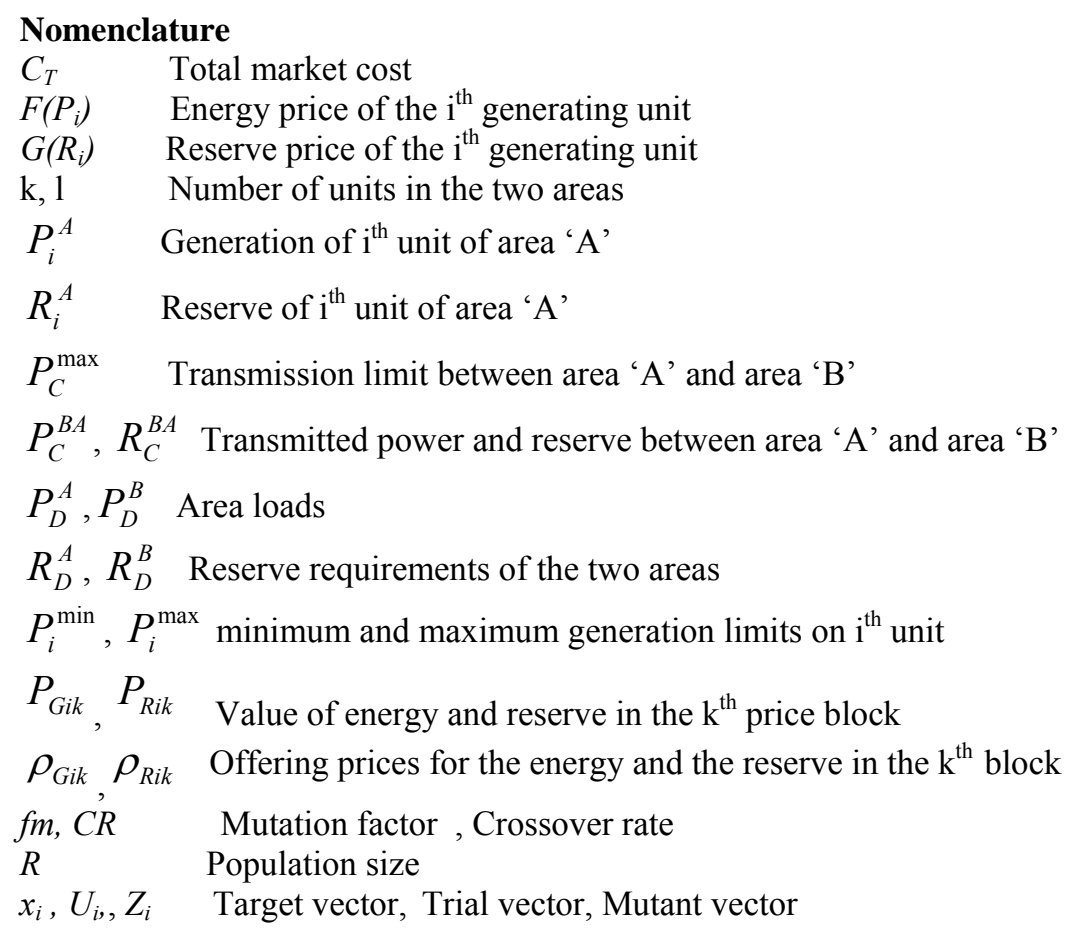

\section{Biographical notes}

Kirti Pal obtained her M.E. degree in Electrical Engineering from M.I.T.S., Gwalior, (India) in 2006 and is presently with Radha Goving Group of Institutions, Meerut, India. She is also perusing her Ph.D. from Rajiv Gandhi Technical University, Bhopal, India. Her research interests are Power System Restructuring and Fuzzy Neural applications to Power System.

M. Pandit obtained her M.Tech degree in Electrical Engineering from Maulana Azad College of Technology, Bhopal, (India) in 1989 and Ph.D. degree from Jiwaji University Gwalior (India) in 2001. She is currently working as Professor in Department of Electrical Engineering, M.I.T.S., Gwalior, (India). Her areas of interest are Power System Security Analysis, Optimization using soft computing/ evolutionary methods, ANN and Fuzzy neural applications to Power System. 
L. Srivastava obtained her M. Tech. degree in Electrical Engineering from the Indian Institute of Technology, Kanpur, India in 1990 and her Ph.D. degree from University of Roorkee (Presently IIT Roorkee), Roorkee, India in 1998. She is working as a Professor and Head in the Department of Electrical Engineering, M.I.T.S. Gwalior, India. She is currently involved in research in power system optimization and control, security analysis, operation and control of deregulated power systems, and ANN and fuzzy logic applications to power system.

Received February 2011

Accepted March 2011

Final acceptance in revised form April 2011 